\title{
Obsessive-Compulsive Inventory-Revised (OCI-R) und Hamburger Zwangsinventar-Kurzform (HZI-K) im Vergleich: Eine Validitätsstudie
}

\author{
Matthias Backenstrass ${ }^{a, b}$ Patrick Schaller ${ }^{c}$ Britta Jäntsch ${ }^{b}$ \\ ${ }^{a}$ Institut für Klinische Psychologie, Zentrum für Seelische Gesundheit, Klinikum Stuttgart, \\ ${ }^{b}$ Klinik für Allgemeine Psychiatrie, Zentrum für Psychosoziale Medizin, Universitätsklinikum Heidelberg, \\ ${ }^{c}$ Psychologisches Institut der Universität Heidelberg, Deutschland
}

\section{Schlüsselwörter}

Zwangsstörung · Konvergente Validität · Diskriminante Validität · Obsessive-Compulsive Inventory-Revised (OCI-R) . Hamburger Zwangsinventar-Kurzform (HZI-K) .

Yale-Brown Obsessive-Compulsive Scale (Y-BOCS)

\section{Zusammenfassung}

Hintergrund: Das Hamburger Zwangsinventar-Kurzform ( $\mathrm{HZI}-\mathrm{K})$ ist ein im deutschen Sprachraum weit verbreitetes Selbstberichtsinstrument zur Erfassung des Schweregrads und der Symptomatik von Zwangsstörungen. Das Obsessive-Compulsive Inventory-Revised (OCl-R), das sich im englischen Sprachraum gut etabliert hat und inzwischen auch in einer deutschsprachigen Version vorliegt, dient ebenfalls der dimensionalen Erfassung von Zwangssymptomen. Die vorliegende Untersuchung hat zum Ziel, einen Vergleich der beiden Instrumente im Hinblick auf Reliabilität und konvergente sowie diskriminante Validität zu liefern. Methode: Basierend auf einer Stichprobe von 41 Patienten mit Zwangsstörungen wurden Korrelationen zwischen $\mathrm{OCl}$ $\mathrm{R}, \mathrm{HZI}-\mathrm{K}$ und anderen Instrumenten zu zwangsrelevanten Konstrukten sowie zur Depression berechnet. Ergebnisse: Die Korrelationen des OCl-R mit dem HZI-K lagen in einem moderaten bis hohen Bereich, während die Korrelationen mit der fremdeingeschätzten Symptomschwere unerwartet niedrig ausfielen. Die Korrelationen zu den Depressionsmaßen lagen mit leicht erhöhten Werten in einem akzeptablen Bereich. Schlussfolgerungen: Insgesamt lieferten die Ergebnisse Belege für eine gute bis sehr gute konvergente Validität des $\mathrm{OCl}-\mathrm{R}$ und $\mathrm{HZI}-\mathrm{K}$. Die beiden Instrumente erwiesen sich somit in vielen Aspekten als vergleichbar. Differenzen bestehen in der Anzahl der Items, den Symptombereichen der Subskalen und der diskriminanten Validität zur Depression. Zusammengefasst lässt sich für die Mehrzahl der Fälle eine systematische Anwendung des $\mathrm{OCl}-\mathrm{R}$ ableiten.

\section{Keywords}

Obsessive-compulsive disorder - Convergent validity . Discriminant validity - Obsessive-Compulsive InventoryRevised (OCl-R) · Hamburg Obsessive-Compulsive Inventory (HZI-K) · Yale-Brown Obsessive-Compulsive Scale (Y-BOCS)

\section{Summary}

A Comparison of the German Version of the ObsessiveCompulsive Inventory-Revised (OCl-R) and the Short Version of the Hamburg Obsessive-Compulsive Inventory (HZI-K): A Validity Study

Background: In Germany, the Hamburg Obsession-Compulsion Inventory ( $\mathrm{HZI}-\mathrm{K}$ ) is widely used as a self-report measure for assessing the symptoms and the severity of obsessive-compulsive disorder (OCD). The Obsessive-Compulsive Inventory-Revised (OCl-R) can also be used to assess symptoms and severity of OCD. It is well established in Englishspeaking countries and is meanwhile also available in German. This study aims to compare these two instruments regarding their reliability and convergent validity, as well as their discriminant validity. Method: Based on a sample of 41 OCD patients, correlations were calculated between OCI-R, $\mathrm{HZI-K}$ and further instruments, assessing OCD-relevant constructs and depression. Results: Correlations between $\mathrm{OCl}-\mathrm{R}$ and $\mathrm{HZI}-\mathrm{K}$ were moderate to high while correlations with observer-rated severity of symptoms were unexpectedly low. Correlations to depression measures were acceptable with slightly raised scores. Conclusions: Overall, the results provided evidence for a good to very good convergent validity of the $\mathrm{OCl}-\mathrm{R}$ and $\mathrm{HZI}-\mathrm{K}$. Both instruments were comparable in many aspects. There are differences concerning the number of items, the symptom areas of the subscales, and the discriminant validity regarding depression. In sum, a systematic application of the OCl-R can be deduced for the majority of cases.

\section{KARGER \\ Fax +497614520714 \\ Information@Karger.de}

www.karger.com (c) 2012 S. Karger GmbH, Freiburg

$1016-6262 / 12 / 0222-0106 \$ 38.00 / 0$

Accessible online at:

www.karger.com/ver
Prof. Dr. phil. Matthias Backenstraß

Institut für Klinische Psychologie

Zentrum für Seelische Gesundheit

Tunzhofer Straße 14-16, 70191 Stuttgart, Deutschland

Tel. +49 711 27822-900, Fax -915

M.Backenstrass@klinikum-stuttgart.de 


\section{Hintergrund}

Zwangsstörungen gehören mit einer 1-Jahres-Prävalenz von $1,2 \%$ und einer Lebenszeitprävalenz von 2,3\% [Ruscio et al., 2010] zu den häufigsten psychischen Störungen. Sie gehen mit einem hohen Leidensdruck und starker Beeinträchtigung im Alltag und der allgemeinen Lebensqualität einher [Eisen et al., 2006]. Diagnostisch wird unter dem Begriff der Zwangsstörung eine Vielzahl relativ unterschiedlicher Symptomkonstellationen zusammengefasst, sodass es sich bei dieser Störungskategorie um eine sehr heterogene Gruppe handelt [Lochner und Stein, 2003]. Dadurch ergibt sich die Notwendigkeit einer - über die rein diagnostische Einordnung hinausreichenden - differenzierten Erfassung der Symptomatik durch psychometrisch gut evaluierte Instrumente [s. dazu beispielsweise Emmelkamp, 2004].

International und auch im deutschen Sprachraum hat sich zur Beurteilung des Schweregrades der Zwangsstörung die Yale-Brown Obsessive Compulsive Scale (Y-BOCS) (deutsche Übersetzung von Hand und Büttner-Westphal [1991]; Originalversion von Goodman et al. [1989]) als Fremdbeurteilungsinstrument durchgesetzt. Im Bereich der Selbstbeurteilungsinstrumente gibt es international eine größere Vielfalt. Neben der Selbstberichtsversion der Y-BOCS [Baer, 1993] ist im deutschen Sprachraum das Hamburger Zwangsinventar (HZI) [Zaworka et al., 1983] vor allem in seiner Kurzform [Klepsch et al., 1993], unter anderem wegen der verfügbaren Normwerte einer klinischen Stichprobe, verbreitet. Das HZI-K besteht aus 72 Items, die das gesamte Kontinuum von Alltagsgewohnheiten bis zu schweren Zwangssymptomen erfassen [Hoyer und Margraf, 2003]. Die Items werden im Forced-Choice-Format («stimmt»/«stimmt nicht») im Selbstbericht beantwortet. Je 12 Items werden zu 6 Unterskalen gruppiert («Kontrollieren», «Waschen, Reinigen», «Ordnen», «Zählen, Berühren, Sprechen», «Denken von Worten und Bildern» und «Gedanken, sich selbst oder anderen ein Leid zuzufügen»), deren Werte durch Aufsummieren der zugehörigen Items berechnet werden. Die psychometrischen Eigenschaften des HZI-K sind mit Werten von Cronbachs $\alpha$ zwischen 0,71 und 0,79 (Ausnahme Skala «Denken von Worten und Bildern» mit $\alpha=0,50)$ sowie Test-Retest-Reliabilitätskoeffizienten zwischen $r_{t t}=0,73$ und 0,94 als zumindest zufriedenstellend für die klinische Anwendung in der Einzelfalldiagnostik zu bezeichnen. Validitätsanalysen weisen auf eine befriedigende diskriminante und eine hohe konvergente Validität hin [Klepsch et al., 1993]. Aber selbst die Kurzform des HZI besteht aus 72 Items, die für Zwangspatienten mit ausgeprägtem Perfektionismus, damit einhergehenden Entscheidungsschwierigkeiten und Rückversicherungszwängen oft nur schwierig und nicht in einem akzeptablen Zeitrahmen zu bearbeiten sind.

Mit dem Obsessive-Compulsive Inventory-Revised (OCIR) legten Foa et al. [2002] eine revidierte Fassung des Obsessive-Compulsive Inventory (OCI) [Foa et al., 1998] vor. Die ursprüngliche Version des OCI umfasst 42 Items, anhand derer insgesamt 7 Subskalen mit unterschiedlichen Symptomdimensionen reliabel und valide erhoben werden können. Zur besseren Anwendbarkeit bei Patienten mit Zwangsstörungen waren Foa et al. [2002] daran interessiert, ein kürzeres und von den psychometrischen Eigenschaften her ebenfalls sehr gutes Erhebungsinstrument zu entwickeln. Die revidierte Fassung (OCI-R) des ursprünglichen Inventars wurde auf insgesamt 18 Items gekürzt. Jeweils 3 Items werden nun zu einer von 6 Unterskalen zusammengefasst: «Kontrollieren», «Waschen», «Ordnen», «Horten», «Mentales Neutralisieren» und «Zwangsgedanken». Zusätzlich wird ein Gesamtwert über alle Items berechnet.

Die autorisierte deutsche Übersetzung des OCI-R erfolgte durch Gönner et al. [2007, 2009]. Die internen Konsistenzen der Subskalen des OCI-R liegen bei Zwangspatienten zwischen Cronbachs $\alpha=0,69$ und $\alpha=0,93$ und für die Gesamtskala zwischen $\alpha=0,81$ und $\alpha=0,84$ [Abramowitz und Deacon, 2006; Foa et al., 2002; Huppert et al., 2007] und damit im befriedigenden bis guten Bereich. Eine Ausnahme bildet die Skala «Mentales Neutralisieren» mit einem Wert von Cronbachs $\alpha=0,57$ [Huppert et al., 2007]. Die Test-Retest-Reliabilität über 2 Wochen lag bei Foa et al. [2002] in der Stichprobe der Zwangspatienten zwischen $r_{t t}=0,74$ und $r_{t t}=0,91$ für die Subskalen und bei $r_{t t}=0,82$ für die Gesamtskala. Die Reliabilitätswerte für die deutsche Version des OCI-R sind mit denen der Originalversion sehr gut vergleichbar, beispielsweise berichten Gönner et al. [2008] Werte zwischen Cronbachs $\alpha=0,76$ und $\alpha=0,95$ für die Subskalen, für die Gesamtskala ergab sich ein Wert von $\alpha=0,85$.

Neben der zufriedenstellenden bis sehr guten Reliabilitätswerte ergaben sich in einer Reihe von Untersuchungen sowohl für die englischsprachige Originalversion als auch für die deutsche Übersetzung überzeugende Hinweise auf die faktorielle, die konvergente und die diskriminante Validität des OCI-R [Übersicht in Gönner et al., 2009]. So wurde beispielsweise die Faktorenstruktur wiederholt durch konfirmatorische Faktorenanalysen empirisch belegt [Gönner et al., 2007, 2008]. Korrelationen zwischen den Skalen des OCI-R und anderen Selbstbeurteilungsskalen zur Erhebung von Zwangssymptomen lagen im mittleren bis hohen Bereich, wie beispielsweise zwischen dem Maudsley Obsessive-Compulsive Inventory (MOCI) [Hodgson und Rachman, 1977] und dem OCI-R zwischen $r=0,56$ und $r=0,85$ [Foa et al., 2002; verschiedene Stichproben; Hajcak et al., 2004]. Gönner et al. [2007] berichten eine Korrelation in Höhe von $r=0,84$ zwischen der Gesamtskala des OCI-R und dem Padua InventoryRevised [PI-R; van Oppen et al., 1995]. Im Hinblick auf die Beziehung zu zwangsrelevanten Konstrukten zeigten sich z.B. in der Studie von Ecker und Gönner [2008] Korrelationen zwischen dem Obsessive-Compulsive Trait Core Dimensions Questionnaire (OC-TCDQ; deutsche Übersetzung durch Ecker und Gönner [2006]; Originalversion von Summerfeldt et al., [2001]) und dem Gesamtwert des OCI-R in Höhe von r 
= 0,69 für die Skala «Unvollständigkeit» bzw. $r=0,48$ für die Skala «Schadensvermeidung», was ebenfalls auf gute bis sehr gute konvergente Validität hinweist.

Zur diskriminanten Validität des OCI-R fanden sich beispielsweise Korrelationen zum State-Trait Anxiety Inventory - Trait Version [Spielberger et al., 1983] in Höhe von $r=0,47$ [Abramowitz und Deacon, 2006] oder zum Penn State Worry Questionnaire (PSWQ) [Meyer et al., 1990] in Höhe von $\mathrm{r}=$ 0,42 [Hajcak et al., 2004; nichtklinische Stichprobe]. Lediglich in Bezug auf Maße zur Depression fanden sich nur akzeptable Werte: Korrelationen zum Beck Depressions-Inventar (BDI) [Beck et al., 1979] lagen über mehrere Studien hinweg zwischen $\mathrm{r}=0,39$ und $\mathrm{r}=0,70$ [Abramowitz und Deacon, 2006; Foa et al., 2002; Hajcak et al., 2004; verschiedene Stichproben].

Ziel der vorliegenden Arbeit ist es, die beiden Selbstbeurteilungsinstrumente HZI-K und OCI-R zur Erhebung von Zwangssymptomatik miteinander zu vergleichen. Das HZI-K ist ein im deutschen Sprachraum sehr etabliertes Verfahren, das OCI-R besticht durch seine Kürze. Es stellt sich die Frage, inwieweit die beiden Instrumente bei einem direkten Vergleich der Symptomskalen via Korrelationsanalysen übereinstimmen und ob sich darüber hinaus im Hinblick auf ihre konvergente und diskriminante Validität weitere Ähnlichkeiten aufzeigen lassen. Quasi als Nebenprodukt sollen mit der vorliegenden Untersuchung die bisherigen Befunde zur Validität des HZI-K und OCI-R um weitere Aspekte ergänzt werden [vgl. dazu beispielsweise Foa et al., 2005].

Aufgrund der sehr ähnlichen Beschreibung von Subskalen beider Instrumente gehen wir davon aus, dass sich hier ein großer Überschneidungsbereich im Sinne hoher korrelativer Zusammenhänge ergibt. Sollte sich die Ähnlichkeit empirisch bestätigen, gehen wir weiter davon aus, dass sich dies auch bei Analysen zur konvergenten und diskriminanten Validität zeigen müsste.

\section{Methodik}

Rahmen der Studie und Vorgehen

Die vorliegende Studie wurde an der Klinik für Allgemeine Psychiatrie des Zentrums für Psychosoziale Medizin des Universitätsklinikums Heidelberg im Rahmen eines größeren Forschungsprojektes durchgeführt. Die Gesamtstudie wurde von der zuständigen Ethikkommission des Universitätsklinikums geprüft und genehmigt. Die Patientenbefragung fand zwischen Anfang des Jahres 2007 und Ende des Jahres 2009 statt. Es wurden sowohl stationäre Patienten der Heidelberger Klinik und des Psychiatrischen Zentrums Nordbaden in Wiesloch als auch ambulante Patienten mit einer Zwangsstörung in die Studie aufgenommen.

Teilnehmende Patienten erhielten Informationen über einige wichtige Eckdaten zum Projekt und eine Einverständniserklärung zur Unterzeichnung. Die Befragung der Patienten fand in Form eines mehrstündigen Interviews in den Räumen der Kliniken statt (unter anderem strukturiertes Interview nach DSM-IV (diagnostisches und statistisches Manual psychischer Störungen), Achse-I und teilweise Achse-II (Zwanghafte Persönlichkeitsstörung und Borderline-Persönlichkeitsstörung), Y-BOCS und anderen). Die Selbstberichtsfragebögen (s.u.) wurden im Regelfall von den Patienten eigenständig - wenn nötig jedoch mit Hilfe der Interviewer - bearbeitet. Voraussetzung für die Teilnahme an der Studie war die Diagnose einer Zwangsstörung nach DSM-IV und ein Y-BOCS-Wert von $\geq 16$.

\section{Stichprobe}

Insgesamt erfüllten $\mathrm{N}=41$ Patienten die Einschlusskriterien, 14 davon waren männlich (34\%) und 27 weiblich (66\%). Das durchschnittliche Alter lag bei $\mathrm{M}=33,44$ Jahren (Standardabweichung $(\mathrm{SD})=9,79$ ). Das Ersterkrankungsalter in Bezug auf die Zwangsstörung lag bei einem Mittelwert von $\mathrm{M}=19,17$ Jahren $(\mathrm{SD}=7,97)$ und die bisherige Dauer der Zwangserkrankung bei einem Mittelwert von $\mathrm{M}=14,15$ Jahren (SD = 10,16). 1 Patient besaß keinen bzw. einen Sonderschulabschluss (2\%), 6 Patienten einen Volks-/Hauptschulabschluss (15\%), 21 Patienten mittlere Reife/Fachschulreife (51\%) und 13 Patienten hatten Abitur/Fachhochschulreife (32\%). 25 Patienten waren berufstätig (61\%), 10 Patienten arbeitslos (24\%), 5 Patienten waren Schüler oder Studenten (12\%) und 1 Patient befand sich in Rente (2\%).

Hinsichtlich der Komorbidität ergaben sich folgende Diagnosen: $8 \mathrm{~Pa}$ tienten erfüllten die Kriterien einer derzeitigen Major Depression (20\%) nach DSM-IV, 4 Patienten erfüllten neben der Zwangsstörung die Kriterien einer Agoraphobie ohne Panikstörung (10\%), 2 die einer Panikstörung mit Agoraphobie (5\%), 5 die einer Sozialen Phobie (12\%), 1 Patient die einer Spezifischen Phobie (2\%) und 6 Patienten erfüllten die Kriterien einer Zwanghaften Persönlichkeitsstörung (15\%). 24 Patienten $(59 \%)$ nahmen zum Untersuchungszeitpunkt Antidepressiva ein.

\section{Erhebungsinstrumente}

Strukturiertes Klinisches Interview für DSM-IV - Achse I und II [SKID I, II; Wittchen et al., 1997]: Das SKID I dient der Erhebung der im DSMIV (deutsche Bearbeitung durch $\mathrm{Saß}$ et al., [2003]) auf Achse I aufgeführten Störungen und das SKID II der Erhebung der Persönlichkeitsstörungen. In der vorliegenden Studie wurden ausschließlich die Kriterien der Zwanghaften und der Borderline-Persönlichkeitsstörung erfragt.

OCI-R [Foa et al., 2002] (deutsche Version von Gönner et al., [2009]): Ausführliche Beschreibung des Instrumentes bereits in der Einleitung (s.o.).

HZI-K [Klepsch et al., 1993]: Auch das HZI-K wurde eingangs ausführlich beschrieben.

Y-BOCS (deutsche Übersetzung durch Hand und Büttner-Westphal [1991]; Originalversion von Goodman et al. [1989]): Die Y-BOCS erfasst in Form eines halbstrukturierten Interviews auf der Basis einer Symptomcheckliste den quantitativen Schweregrad einer Zwangsstörung, unabhängig von deren konkretem Inhalt. Die Y-BOCS liefert einen Summenwert für Zwangsgedanken und Zwangshandlungen sowie einen Gesamtwert.

Obsessive Beliefs Questionnaire (OBQ) [OCCWG, 2001] (deutsche Version: Ertle et al., [2008]): Das OBQ der Obsessive-Compulsive Cognitions Working Group (OCCWG) dient der Erfassung (im Selbstbericht) zwangstypischer dysfunktionaler Einstellungen. Es besteht aus insgesamt 44 Items, die auf einer Skala von «sehr starke Ablehnung» $(=1)$ bis «sehr starke Zustimmung» (=7) eingeschätzt werden. Die Skalenbildung haben wir entsprechend den Faktorenanalysen von Ertle et al. [2008] vorgenommen, die eine Zuweisung der Items zu den 3 Skalen «Bedeutsamkeit von Gedanken/Notwendigkeit, Gedanken zu kontrollieren», «Perfektionismus/Unsicherheitsintoleranz» und «Gefahrenüberschätzung/Überhöhte subjektive Verantwortlichkeit» sowie einen Gesamtwert vorgeschlagen haben. Cronbachs alpha ( $\alpha=0,81$ bis 0,92 für eine klinische Stichprobe) sowie Test-Retest-Reliablitäts-Koeffizienten ( $\mathrm{r}=0,71$ bis 0,83 für eine nichtklinische Stichprobe) sind für diese Skalenbildung als befriedigend bis gut einzuschätzen [Ertle et al., 2008]. Hinweise auf die Validität ergeben sich sowohl für die deutsche [Ertle et al., 2008] als auch die Originalversion [OCCWG, 2005].

OC-TCDQ (deutsche Übersetzung durch Ecker und Gönner [2006]; Originalversion von Summerfeldt et al. [2001]): Das OC-TCDQ ist ein Instrument zur Erfassung (im Selbstbericht) der beiden Dimensionen Un- 
vollständigkeit und Schadensvermeidung, von denen vermutet wird, dass sie zentrale motivationale Aspekte der Zwangsstörungen bilden (deshalb «Kerndimensionen») [s. beispielsweise Summerfeldt, 2004]. Das OCTCDQ besteht aus insgesamt 20 Items, von denen jeweils $10 \mathrm{zu}$ einer der beiden Dimensionen zusammengefasst werden. Reliabilitätsanalysen erbrachten mit $\alpha=0$,91 für Schadensvermeidung und $0,90 \leq \alpha \leq 0,93$ für Unvollständigkeit [Coles et al., 2005; Ecker und Gönner, 2008; Pietrefesa und Coles, 2008] sehr gute Werte für die beiden Subskalen. Auch Belege für die konvergente Validität und Konstruktvalidität liegen vor (deutsche Version: Ecker und Gönner [2008]; englische Version: Pietrefesa und Coles [2008]).

BDI [Beck et al., 1979] (deutsche Version: Hautzinger et al. [1994]): Das BDI ist ein Selbstberichtsinstrument, das der quantitativen Erfassung des Schweregrades einer Depression dient.

Hamilton-Depressionsskala [HAM-D; Hamilton, 1960; deutsche Version: Collegium Internationale Psychiatriae Scalarum, 1996]: Die HAM-D ist ein Fremdeinschätzungsinstrument zur Beurteilung der Schwere einer Depression.

\section{Ergebnisse}

\section{Interne Konsistenzen von HZI-K und OCI-R}

Der niedrigste Cronbachs $\alpha$-Wert für die Skalen des OCI-R wurde für «Mentales Neutralisieren» $(\alpha=0,69)$ ermittelt, beim HZI-K lag der niedrigste Wert bei $\alpha=0,71$ ( Denken von Worten und Bildern»). Die übrigen Werte für die Subskalen des OCI-R lagen in einem guten bis sehr guten Bereich $(0,82 \leq \alpha \leq 0,91)$, für den Gesamtwert ergab sich dagegen ein etwas niedriger Koeffizient $(\alpha=0,71)$. Für das HZI-K lag Cronbachs $\alpha$ für den Gesamtwert etwas höher $(\alpha=0,87)$, dagegen erwies sich neben der Skala «Denken von Worten und Bildern» $(\alpha=0,71)$ die Dimension «Zählen, Berühren, Sprechen» $(\alpha=0,72)$ als weniger reliabel (Range auf Dimensionsebene insgesamt: $0,71 \leq \alpha \leq 0,89)$.

\section{Konvergente Validität}

\section{Interkorrelationen der Symptomdimensionen von HZI-K und} $O C I-R$

Wie Tabelle $1 \mathrm{zu}$ entnehmen ist, ergaben sich auf dem $1 \%$-Niveau statistisch signifikante Korrelationskoeffizienten im Wertebereich von $r=0,56$ bis $r=0,85$ zwischen den HZIK-Skalen und den OCI-R-Skalen, die von der Skalenbezeichnung her vorgeben, gleiche Symptomdimensionen zu erheben. Die HZI-K-Skala «Denken von Worten und Bildern» hat ihre Entsprechung am ehesten in der OCI-R-Skala «Zwangsgedanken», wenn auch ein $r=0,36$ darauf hindeutet, dass die Skalen eher wenig Gemeinsames messen. Da die OCI-R-Skala «Zwangsgedanken» auch mit «Gedanken, sich selbst oder anderen ein Leid zuzufügen» des HZI-K signifikant korrelierte, kann angenommen werden, dass das HZI-K mit den beiden Skalen «Denken von Worten und Bildern» und «Gedanken, sich selbst oder anderen ein Leid zuzufügen» Zwangsgedanken differenzierter abbildet. Dass die OCI-R-

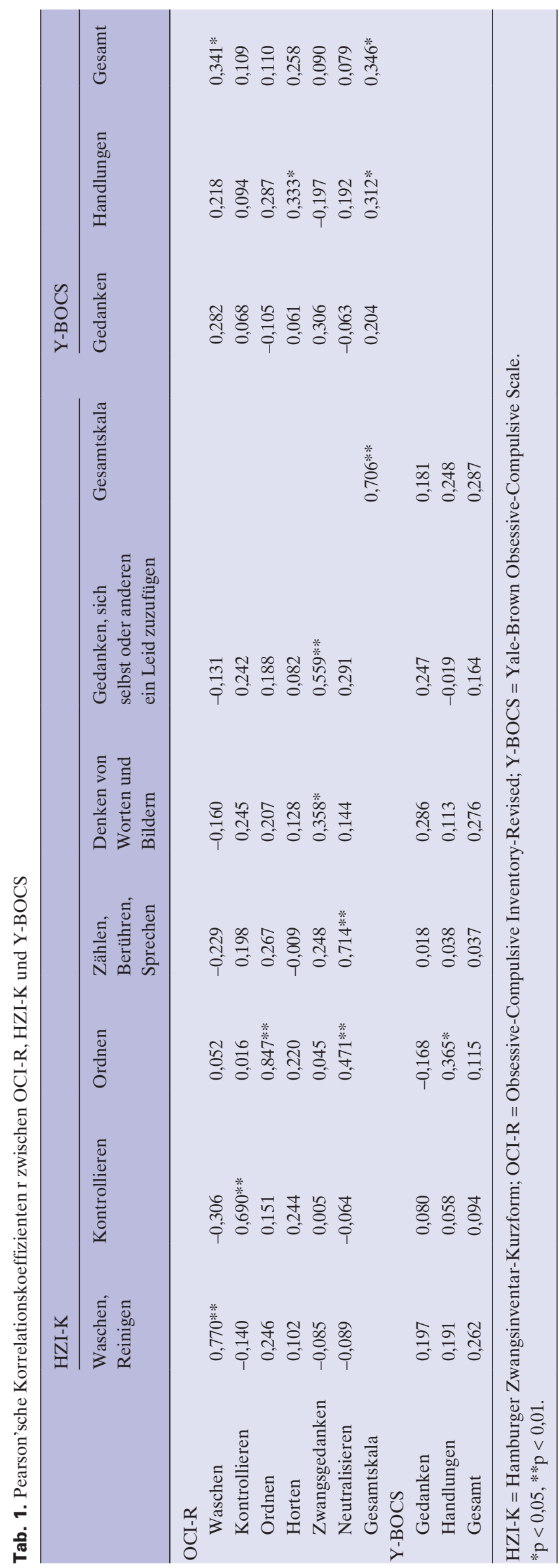

Verhaltenstherapie 2012;22:106-113 
Skala «Horten» keine Entsprechung im HZI-K hat, wird auch durch die statistisch nicht bedeutsamen Korrelationen zwischen «Horten» und den Symptomdimensionen des HZI-K bestätigt. Die Gesamtwerte der beiden Skalen korrelierten ebenfalls hochsignifikant miteinander.

\section{Korrelationen von HZI-K und OCI-R mit der Y-BOCS}

Die Korrelation zwischen dem Gesamtwert der Y-BOCS und HZI-K erwies sich als nicht signifikant (vgl. Tab. 1). Der OCIR-Gesamtwert korrelierte dagegen statistisch bedeutsam mit dem Y-BOCS-Gesamtwert. Von den Symptomdimensionen des OCI-R korrelierte keine signifikant mit der Y-BOCSZwangsgedankenskala. Y-BOCS-Zwangshandlungen korrelierten signifikant nur mit der OCI-R-Skala «Horten». Von den Symptomdimensionen des HZI-K korrelierte lediglich «Ordnen» signifikant mit den Y-BOCS-Zwangshandlungen (vgl. Tab. 1).

\section{HZI-K, OCI-R und zwangsrelevante Konstrukte}

Eine sehr ähnliche Erfassung der Zwangssymptomatik durch die beiden Erhebungsinstrumente OCI-R und HZI-K sollte sich nicht nur in hohen Interkorrelationen gleicher Subskalen, sondern auch in ähnlichen Korrelationsmustern zu anderen Konstrukten bzw. deren Operationalisierungen abbilden. In Tabelle 2 sind die Korrelationen der OCI-R- und HZI-KWerte mit den Skalenwerten des OBQ und des OC-TCDQ dargestellt. Wie in Tabelle 2 zu sehen ist, ergaben sich sehr ähnliche Korrelationsmuster zwischen den Skalen des OBQ einerseits sowie den HZI-K- und OCI-R-Gesamtwerten andererseits. Auch für die Subskalen der beiden Zwangssymptomatik-Instrumente «Waschen», «Kontrollieren» und «Ordnen» ergaben sich sehr ähnliche Korrelationsmuster (vgl. Tab. 2). Weniger einheitlich fielen die Korrelationsmuster der übrigen Symptomskalen von HZI-K («Zählen, Berühren, Sprechen», «Denken von Worten und Bildern» und «Gedanken, sich selbst oder anderen ein Leid zuzufügen») und OCI-R («Zwangsgedanken», «Horten» und «Neutralisieren») aus, die sich bereits von der Konzeptbildung her und auch von den weiter oben berichteten Interkorrelationen unterschieden.

Schließlich waren auch die Zusammenhänge zwischen den als Kernmotivationen für Zwangssymptome angenommenen «Schadensvermeidung» und «Unvollständigkeitsgefühl» einerseits und den beiden Symptomatik-Instrumenten andererseits sehr ähnlich: Die Gesamtwerte von OCI-R und HZI-K korrelierten beide hochsignifikant mit den beiden Skalen des OC-TCDQ «Schadensvermeidung» und «Unvollständigkeit» in positiver Richtung (erhöhte Symptomatik ging mit einer stärkeren Ausprägung der beiden Kernmotivationen einher). Darüber hinaus zeigten die Subskalen «Waschen», «Ordnen» und «Kontrollieren» beider Instrumente nahezu identische Korrelationsmuster. Sowohl HZI-K als auch OCI-R «Ordnen» wiesen einen statistisch signifikanten Zusammenhang zum Unvollständigkeitserleben jedoch nicht zur Schadensvermeidung auf (Tab. 2).

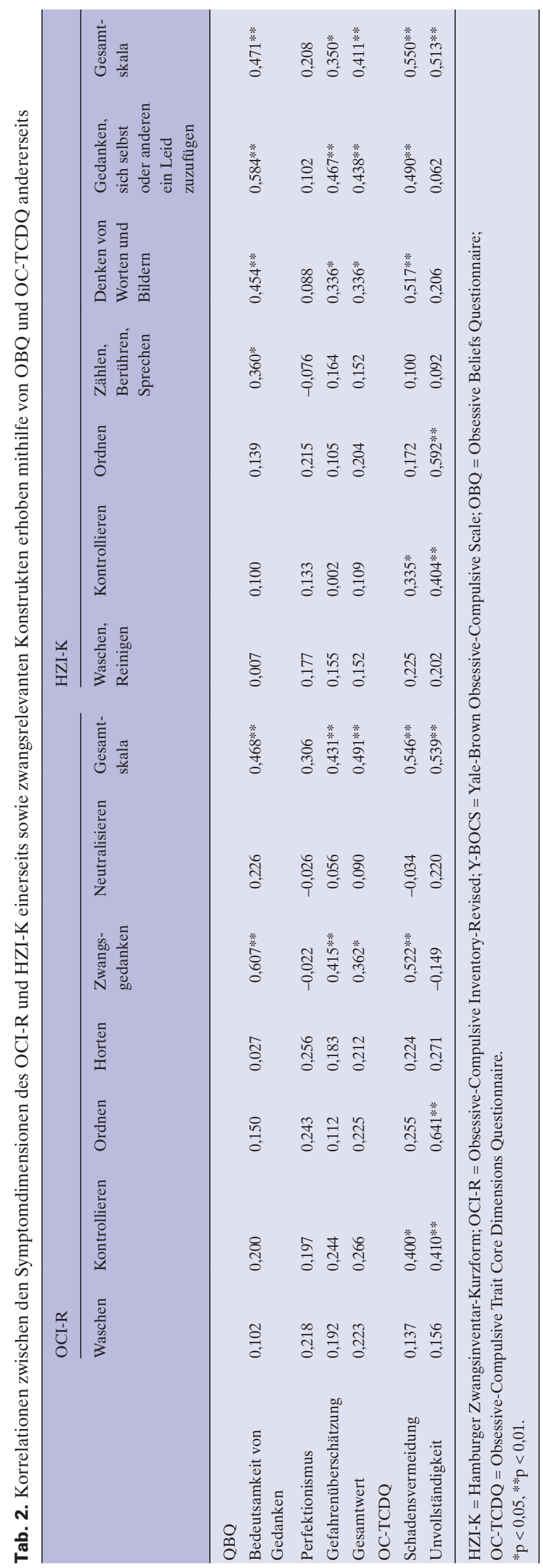

Backenstrass/Schaller/Jäntsch 
Tab. 3. Korrelationen des OCI-R und HZI-K mit BDI und HAM-D

\begin{tabular}{|c|c|c|c|c|c|c|c|}
\hline HZI-K & $\begin{array}{l}\text { Waschen, } \\
\text { Reinigen }\end{array}$ & Kontrollieren & Ordnen & $\begin{array}{l}\text { Zählen, } \\
\text { Berühren, } \\
\text { Sprechen }\end{array}$ & $\begin{array}{l}\text { Denken von Worten } \\
\text { und Bildern }\end{array}$ & $\begin{array}{l}\text { Gedanken, sich } \\
\text { selbst oder } \\
\text { anderen ein Leid } \\
\text { zuzufügen }\end{array}$ & Gesamtskala \\
\hline BDI & 0,193 & 0,079 & 0,200 & $-0,178$ & 0,025 & 0,018 & 0,124 \\
\hline HAM-D & 0,121 & $-0,155$ & $0,436 * *$ & 0,065 & $-0,066$ & 0,185 & 0,193 \\
\hline OCI-R & Waschen & Kontrollieren & Ordnen & Horten & Zwangsgedanken & Neutralisieren & Gesamtskala \\
\hline BDI & 0,259 & $-0,091$ & $0,487 * *$ & 0,114 & 0,155 & 0,306 & $0,410^{*}$ \\
\hline HAM-D & 0,094 & 0,170 & $0,329^{*}$ & $0,410 * *$ & $-0,025$ & 0,055 & $0,354^{*}$ \\
\hline
\end{tabular}

\section{Diskriminante Validität}

Dass die Korrelationen der Subskalen des HZI-K und des OCI-R, die von der Benennung und den zugrunde gelegten Symptomgruppen her unterschiedliche Beschwerden zusammenfassen, alle statistisch nicht signifikant waren (s. Tab. 1, Ausnahme HZI-K «Ordnen» und OCI-R «Neutralisieren»), kann als erster Beleg im Sinne der diskriminanten Validität der Symptomdimensionen verstanden werden.

\section{HZI-K, OCI-R und Depression}

Bisherige Validitätsstudien zum OCI-R haben sich auf Depressionsmaße, die im Rahmen einer Selbstbeurteilung erhoben wurden, gestützt. In Tabelle 3 ist die durch Selbstbeurteilung erhobene Depressivität ergänzt durch eine Rater-gestützte Erhebung (HAM-D) des Schweregrades depressiver Symptomatik dargestellt. Der Gesamtwert des HZI-K korrelierte nicht signifikant mit den beiden Depressionsmaßen. Von den HZI-K-Subskalen korrelierte ausschließlich die Dimension «Ordnen» mit der fremdbeurteilten Depressivität statistisch signifikant, und zwar dahingehend, dass höhere Depressionswerte mit höheren Werten auf der Dimension «Ordnen» assoziiert waren. Auch die «Ordnen»-Dimension des OCI-R korrelierte statistisch signifikant mit der Depressivität, sowohl in der Fremd- als auch in der Selbstbeurteilung (Tab. 3). Darüber hinaus ergaben sich signifikante Korrelationen zwischen dem OCI-R-Gesamtwert und den Depressionsmaßen.

\section{Diskussion}

Ziel der vorliegenden Untersuchung war es, die beiden Selbstbeurteilungsinstrumente HZI-K und OCI-R, die beide der Erhebung der Schwere von Zwangssymptomen dienen, miteinander zu vergleichen. Der Vergleich wurde im Hinblick auf Reliabilität, konvergente und diskriminante Validität durchgeführt. Sowohl im Vergleich zu der deutschen Version als auch der englischen Originalversion des OCI-R zeigte sich die interne Konsistenz für die Gesamtskala des OCI-R in unserer Studie vor dem Hintergrund, dass das OCI-R wie auch das HZI-K zur Individualdiagnostik eingesetzt wird, als weniger befriedigend im Vergleich zu den bisher publizierten Werten [vgl. Foa et al., 2002; Gönner et al., 2009]. Die interne Konsistenz für den Gesamtwert des HZI-K erwies sich als zufriedenstellender. Trotz der deutlich geringeren Anzahl an Items pro Subskala zeigten sich die internen Konsistenzen für die Symptomdimensionen des OCI-R bis auf «Mentales Neutralisieren» als sehr zufriedenstellend und nicht schlechter als die Werte für die HZI-K-Symptomdimensionen. Vor dem Hintergrund von in der Literatur berichteten Retest-Reliabilitäten für das OCI-R und das HZI-K kann für beide Instrumente von befriedigenden bis sehr guten Reliabilitäten ausgegangen werden.

Im direkten Vergleich der beiden Instrumente bezüglich ihrer Subskalen ist festzustellen, dass sie in den konzeptionell sehr ähnlichen Skalen «Waschen», «Kontrollieren» und «Ordnen» sehr hohe Interkorrelationen zeigen. Noch etwas höhere Werte berichten Gönner et al. [2009] für entsprechende Korrelationen zwischen Subskalen des OCI-R und des PI-R, die die konvergente Validität des OCI-R bestätigen. Im Gegensatz zu den Ergebnissen, die mit der englischen Originalversion des OCI-R ermittelt wurden [Foa et al., 2002], ergaben sich in der vorliegenden Untersuchung geringe Korrelationen zwischen den Y-BOCS-Werten und den OCI-R-Skalen. Interessant in diesem Zusammenhang ist, dass Gönner et al. [2007, 2008] mit der Selbstberichtsversion der Y-BOCS zwar höhere Werte als die hier vorgestellten berichten. Aber auch bei Gönner et al. fielen die Korrelationen der OCI-R-Skalen mit der Zwangsschwere (Y-BOCS) im Vergleich zum englischen Original geringer aus. Der Zusammenhang des HZI-K-Gesamtwertes mit den Y-BOCS-Skalen erwies sich in unserer Studie als statistisch nicht signifikant. Die Ergebnisse unserer und der zitierten Studien bestätigen die unterschiedlichen Konzeptionen der Y-BOCS einerseits (sei es Selbst- oder FremdratingVersion) und des OCI-R sowie HZI-K andererseits. Während 
letztere Instrumente entwickelt wurden, um vor allem unterschiedliche Zwangssymptomdimensionen zu erfassen, misst die Y-BOCS, unabhängig von der jeweils spezifischen Symptomatik, den Schweregrad der Störung und berücksichtigt dabei sehr viel stärker Aspekte wie beispielsweise Leidensdruck und Widerstand gegen die Zwangssymptomatik.

Die vergleichende Analyse der konvergenten Validität von HZI-K und OCI-R führt in Bezug auf dysfunktionale Einstellungen, wie sie mit dem OBQ erhoben werden, zu nahezu identischen Ergebnissen: Ausgeprägtere Zwangssymptomatik geht einher mit höheren Werten in den Skalen «Bedeutsamkeit von Gedanken», «Gefahrenüberschätzung» und der Gesamtausprägung dysfunktionaler Einstellungen. Ähnliche Befunde ermittelten auch Ertle et al. [2008], die bei einer Stichprobe von Zwangspatienten einen signifikanten Zusammenhang zwischen Symptomschwere und «Perfektionismus» fanden, jedoch keine signifikante Korrelation zwischen Symptomschwere und der OBQ-Skala «Bedeutsamkeit von Gedanken». Die sehr gute konvergente Validität von OCI-R und HZI-K zeigte sich auch auf der Ebene der Symptomdimensionen: Die namensgleichen Dimensionen zeigten identische (im Sinne von Richtung und Signifikanz) Korrelationsmuster zwischen Symptomschwere und dysfunktionaler Einstellung. So stehen z.B. Zwangsgedanken (egal, ob mit HZI-K oder OCI-R erhoben) in signifikant positivem Zusammenhang zu «Bedeutsamkeit von Gedanken» und «Gefahrenüberschätzung», jedoch nicht zu «Perfektionismus».

Den Befunden von Ecker und Gönner [2008] entsprechend ermittelten auch wir sowohl mit dem OCI-R als auch anhand des HZI-K positive Zusammenhänge zwischen der Symptomschwere und den als Kernmotivationen vermuteten «Schadensvermeidung» und «Unvollständigkeit»: Je mehr die betroffenen Patienten über Zwangssymptome berichten, desto höher ist die Neigung zur Schadensvermeidung und zum Unvollständigkeitserleben ausgeprägt. Ebenfalls in Übereinstimmung mit dem Modell der Kernmotivationen von Summerfeldt [2004] und den empirischen Befunden von Ecker und Gönner [2008] steht der positive Zusammenhang von «Ordnen» und Unvollständigkeitserleben. Ordnen als Zwangssymptom steht aber nicht in Zusammenhang mit dem Motiv der Schadensvermeidung. Zwangsgedanken dagegen wurden in Verbindung mit Schadensvermeidung gebracht, was ebenfalls mit unseren Befunden in Übereinstimmung steht und sowohl durch die OCI-R- als auch die HZI-K-Operationalisierung bestätigt wird.

Unsere Ergebnisse bestätigen die bereits mit der Originalversion [Foa et al., 2002] als auch mit der deutschen Version ermittelten substanziellen Korrelationen des OCI-R mit Depressionsmaßen [Gönner et al., 2007]: Höhere Depressionswerte gehen mit höheren Werten in der Gesamtskala des OCI-R einher. Diese «Anfälligkeit» hat sich für das HZI-K nicht gezeigt. Möglicherweise immunisiert die einfache Antwortmöglichkeit «stimmt»/«stimmt nicht» des HZI-K im Gegensatz zur 4-stufigen Antwortmöglichkeit des OCI-R gegen den Einfluss eines depressionsbasierten Bias.

Bei der Interpretation unserer Ergebnisse ist zu berücksichtigen, dass die Stichprobengröße relativ gering ist. Die bedeutsamste Folge davon ist, dass mittlere Unterschiede zwischen den ermittelten Korrelationen als nicht signifikant ausgewiesen werden würden, obwohl sie inhaltlich von Bedeutung sein könnten. Wir haben deshalb auf eine systematische Signifikanztestung von Korrelationsunterschieden verzichtet, wie sie z.B. für den Zusammenhang von HZI-K und OCI-R einerseits und den Depressionsmaßen andererseits interessant gewesen wären.

Zusammenfassend lässt sich feststellen, dass beide Instrumente bei hoher konvergenter Validität ihre Vorteile und Nachteile haben. Der besondere Vorteil des OCI-R liegt in seiner Kürze. Zudem wird beim OCI-R die Symptomdimension «Horten» erhoben. Damit empfiehlt sich das OCI-R für eine routinemäßige Anwendung im klinischen Kontext. Die Stärken des HZI-K liegen in einer differenzierteren Erfassung von psychopathologischen Phänomenen im Bereich der Zwangsgedanken und in einer geringeren «Anfälligkeit» gegenüber depressiver Symptomatik. Dem HZI-K kommt damit im Vergleich zum OCI-R nur noch ein eingeschränkter Indikationsbereich zu.

\section{Dank}

Die Autoren danken allen Patienten, die bereit waren, an der Studie teilzunehmen. Darüber hinaus möchten wir Frau Dipl.-Psych. Judith Spannagel, Frau Dipl.-Psych. Laura Laventure und Herrn cand. Psych. Martin Knittel für die Unterstützung bei der Studiendurchführung danken. Schließlich gilt unser Dank Herrn Dr. Olivier Elmer, der uns bei der Patientenrekrutierung im Psychiatrischen Zentrum Nordbaden (PZN) unterstützt hat. Herrn Dr. Sascha Gönner und Herrn Privatdozent Dr. Willi Ecker möchten wir für die Überlassung des OCI-R und des OCTCDQ danken.

\section{Disclosure Statement}

Hiermit erklären die Autoren, dass im Zusammenhang mit dem von ihnen verfassten Artikel keine Interessenkonflikte vorliegen. 


\section{Literatur}

Abramowitz JS, Deacon BJ: Psychometric properties and construct validity of the Obsessive-Compulsive Inventory-Revised: Replication and extension with a

clinical sample. J Anxiety Disord 2006;20:1016-1035

Baer L: Alles unter Kontrolle - Zwangsgedanken und Zwangshandlungen überwinden. Bern, Huber, 1993.

Beck AT, Rush AJ, Shaw BF, Emery G: Cognitive Therapy of Depression. New York, Guilford Press, 1979

Collegium Internationale Psychiatriae Scalarum: Internationale Skalen für Psychiatrie. Göttingen, Beltz, 1996.

Coles ME, Heimberg RG, Frost RO, Steketee G: Not

just right experiences and obsessive-compulsive features: Experimental and self-monitoring perspectives. Behav Res Ther 2005;43:153-167.

Ecker W, Gönner S: Das Unvollständigkeitsgefühl

Neuentdeckung eines alten psychopathologischen Symptoms bei Zwangserkrankungen. Nervenarzt 2006;77:1115-1122.

Ecker W, Gönner S: Incompleteness and harm avoid-

ance in OCD symptom dimensions. Behav Res Ther 2008;46:895-904.

Eisen JL, Mancebo MA, Pinto A, Coles ME, Pagano

ME, Stout R, Rasmussen SA: Impact of obsessivecompulsive disorder on quality of life. Compr Psychiatry 2006:47:270-275.

Emmelkamp PMG: The additional value of clinimet-

rics needs to be established rather than assumed. Psychother Psychosom 2004;73:142-144.

Ertle A, Wahl K, Bohne A, Moritz S, Kordon A,

Schulte D: Dimensionen zwangsspezifischer Einstellungen: Der Obsessive-Beliefs Questionnaire (OBQ) für den Deutschen Sprachraum analysiert. Z Klin Psychol Psychother 2008;37:263-271.

Foa EB, Huppert JD, Leiberg S, Langner R, Kichic R,

Hajcak G, Salkovskis PM: The Obsessive-Compulsive Inventory: Development and validation of a short version. Psychol Assess 2002;14:485-495.

Foa EB, Kozak MJ, Salkovskis P, Coles ME, Amir N

The validation of a new obsessive-compulsive disorder scale: The Obsessive-Compulsive Inventory. Psychol Assess 1998;10:206-214.

Foa EB, Liebowitz MR, Kozak MJ, Davies S,

Campeas R, Franklin ME, Huppert JD, Kjernisted K, Rowan V, Schmidt AB, Simpson HB, Tu X: Randomized, placebo-controlled trial of exposure and ritual prevention, clomipramine, and their combination in the treatment of obsessive-compulsive disorder. Am J Psychiatry 2005;162:151-161.
Gönner S, Ecker W, Leonhart R: Obsessive-Compulsive Inventory - Revised (OCI-R) - Deutsche Adaptation. Manual. Frankfurt, Pearson, 2009.

Gönner S, Leonhart R, Ecker W: Das Zwangsinventar

OCI-R - die deutsche Version des Obsessive-Compulsive Inventory-Revised: Ein kurzes Selbstbeurteilungsinstrument zur mehrdimensionalen Messung von Zwangssymptomen. Psychother Psychosom Med Psychol 2007;57:395-404.

Gönner S, Leonhart R, Ecker W: The Obsessive-Compulsive Inventory-Revised (OCI-R): Validation of the German version in a sample of patients with OCD, anxiety disorders, and depressive disorders. J Anxiety Disord 2008;22:734-749.

Goodman WK, Price LH, Rasmussen SA, Mazure C:

The Yale-Brown Obsessive Compulsive Scale: I. Development, use, and reliability. Arch Gen Psychiatry 1989;46:1006-1011.

Hajcak G, Huppert JD, Simons RF, Foa EB: Psycho-

metric properties of the OCI-R in a college sample. Behav Res Ther 2004;42:115-123.

Hamilton M: A rating scale for depression. J Neurol

Neurosurg Psychiatry 1960;23:56-62.

Hand I, Büttner-Westphal H: Die Yale-Brown Obses-

sive Compulsive Scale (Y-BOCS): Ein halbstrukturiertes Interview zur Beurteilung des Schweregrades von Denk- und Handlungszwängen. Verhaltenstherapie 1991;1:223-225.

Hautzinger M, Bailer M, Worall H, Keller F: BeckDepressions-Inventar (BDI). Bern, Huber, 1994

Hodgson RJ, Rachman S: Obsessional-compulsive complaints. Behav Res Ther 1977;15:389-395.

Hoyer J, Margraf J: Angstdiagnostik. Grundlagen und Testverfahren. Berlin, Springer, 2003.

Huppert JD, Walther MR, Hajcak G, Yadin E, Foa

EB, Simpson HB, Liebowitz MR: The OCI-R: Validation of the subscales in a clinical sample. $\mathrm{J}$ Anxiety Disord 2007;21:394-406.

Klepsch R, Zaworka W, Hand I, Lünenschloß K, Jauernig G: Hamburger Zwangsinventar - Kurzform (HZI-K). Manual. Göttingen, Beltz Test GmbH, 1993.

Lochner C, Stein DJ: Heterogeneity of obsessive-com-

pulsive disorder: A literature review. Harvard Rev Psychiatry 2003;11:113-132.
Meyer TJ, Miller ML, Metzger RL, Borkovec TD: De velopment and validation of the Penn State Worry Questionnaire. Behav Res Ther 1990;28:487-495.

OCCWG: Development and initial validation of the

obsessive beliefs questionnaire and the interpretation of intrusions inventory. Behav Res Ther 2001 39:987-1006.

OCCWG: Psychometric validation of the obsessive be-

lief questionnaire and interpretation of intrusions inventory - Part 2: Factor analyses and testing of a brief version. Behav Res Ther 2005;43:1527-1542.

Pietrefesa AS, Coles ME: Moving beyond an exclusive

focus on harm avoidance in obsessive compulsive disorder: Considering the role of incompleteness. Behav Res Ther 2008;39:224-231.

Ruscio AM, Stein DJ, Chiu WT, Kessler RC: The epi-

demiology of obsessive-compulsive disorder in the National Comorbidity Survey Replication. Mol Psychiatry 2010;15:53-63.

$\mathrm{Sa}$ H, Wittchen H-U, Zaudig M, Houben I: Diagnostisches und statistisches Manual psychischer Störungen. Textrevision. Göttingen, Hogrefe, 2003.

Spielberger CD, Gorsuch RL, Lushene R, Vagg PR, Jacobs GA: Manual for the State-Trait Anxiety Inventory. Palo Alto, Consulting Psychologists Press, 1983.

Summerfeldt LJ: Understanding and treating incom-

pleteness in obsessive-compulsive disorder. J Clin Psychol 2004:60:1155-1168.

Summerfeldt LJ, Kloosterman PH, Parker JDA, Antony MM, Swinson RP: Assessing and validating the obsessive-compulsive-related construct of incompleteness; in: Poster presented at the 62nd Annual Convention of the Canadian Psychological Association. Ste-Foy, Quebec, 2001.

van Oppen P, Hoekstra RJ, Emmelkamp PMG: The

structure of obsessive-compulsive symptoms. Behav Res Ther 1995;33:15-23.

Wittchen H-U, Zaudig M, Fydrich T: Strukturiertes Klinisches Interview für DSM-IV. Göttingen, Hogrefe, 1997.

Zaworka W, Hand I, Jauernig G, Lünenschloß K: Hamburger Zwangsinventar. Fragebogen zur Erfassung von Zwangsgedanken und Zwangsverhalten (HZI). Göttingen, Beltz, 1983. 